\title{
The supraorbital keyhole approach: how I do it
}

\author{
Harjinder Singh Bhatoe ${ }^{1}$
}

Received: 18 June 2015 / Accepted: 18 July 2015 /Published online: 8 August 2015

(C) Springer-Verlag Wien 2015

\section{Dear Editor,}

We read with great interest the article entitled "The supraorbital keyhole approach: how I do it" by Reisch et al. [2]. The article is obviously backed by the huge experience of the authors, and the description is very succinct, and clearly described, step by step.

I have been following this approach since 2003 for the clipping of anterior circulation aneurysms [1], and excision of various tumours, especially those in and around the sellar region (multicompartmental pituitary adenomas, craniopharyngiomas, hypothalamic and chiasmatic gliomas, suprasellar epidermoid), meningiomas of the anterior cranial fossa, orbital space occupying lesions and traumatic cerebrospinal fluid (CSF) leaks through the anterior skull base. While the description of the procedure by the authors is quite specific, concise and clear, I may please be permitted to add the following points:

1. An inferiorly based pericranial flap can be made with success. The flap can be used for dura closure (the dura is often seen to be desiccated towards the end of procedure, despite being covered with wet patties, and tends to tear during closure).

2. The inner table of the skull from the superior margin of the craniotomy defect can be removed with fine Kerrison's rongeurs. This gives a few extra millimetres of space at the inside margin.

3. Saving bone dust and bone chips, and their use during fixing the bone flap, improves postoperative appearance and prevents linear supraciliary defect.

Harjinder Singh Bhatoe

hsbhatoe@gmail.com

1 Max Super Specialty Hospital PPG, 108A IP Extension, New Delhi 110092, Delhi, India
4. The frontal pole is retracted to suction out CSF, and gradually the lobe falls by gravity. It is obvious that wide arachnoid dissection and opening of cisterns holds the key to exposure of the anatomy and defining the pathology.

5. An extremely important and valuable step is opening of the Sylvian fissure, which makes frontal lobe to move away and eases retraction. The Sylvian fissure is opened in every patient irrespective of pathology.

6. Frequent adjustment of retraction (when applied) and movement of microscope gives the necessary leeway and exposure to all the aspects of the pathology.

Authors have already emphasised the need for special tube shaft instruments. The use of bipolar forceps with angled tips is also a valuable adjunct. A neuroendoscope $\left(0^{\circ}\right.$ and $\left.30^{\circ}\right)$ can be used to assess the completion of the procedure.

Last but not the least, a word of caution. The approach should be taken up only when one is familiar with the pterional approach, and has developed the judgment for selection of the right pathology and the right patient. I do not advocate this approach for the beginners. Only when one is familiar with microanatomy, comfortable with the specialised instruments and working through narrow confined spaces, should this approach be adopted, under mentorship. Assimilation of the concept of minimalism in operative neurosurgery is a gradual process, and as with any procedure, this too has its learning curve.

\section{References}

1. Bhatoe HS (2009) Transciliary supraorbital keyhole approach for anterior circulation aneurysms of anterior circulation: operative nuances. Neurol India 57:599-606

2. Reisch R, Marcus HJ, Kockro RA, Ulrich NH (2015) The supraorbital keyhole approach: how I do it. Acta Neurochir (Wein) 157:979-983 